\title{
MicroRNA-140 suppresses Helicobacter pylori-positive gastric cancer growth by enhancing the antitumor immune response
}

\author{
MIN ZHAO ${ }^{1}$, QIAN LIU ${ }^{1}$, WENXIANG LIU ${ }^{2}$, HE ZHOU $^{1}$, XUAN ZANG $^{1}$ and JIANGYANG LU ${ }^{1}$ \\ Departments of ${ }^{1}$ Pathology and ${ }^{2}$ Digestion, The First Affiliated Hospital of \\ General Hospital of People's Liberation Army, Beijing 100048, P.R. China
}

Received April 14, 2018; Accepted November 14, 2018

DOI: $10.3892 / \mathrm{mmr} .2019 .10475$

\begin{abstract}
Immune checkpoint blockade is a promising therapeutic strategy against various human malignancies. MicroRNAs (miRNAs/miRs) regulate gene expression, by repressing mRNA translation or promoting its degradation. The aim of the current study was to investigate the role and molecular mechanisms of miR-140 in Helicobacter pylori (Hp)-associated gastric cancer, and to examine its relationship with immune function in gastric cancer. Gastritis tissue samples from gastritis patients, and gastric cancer tissue samples from gastric cancer patients were collected for miR-140 expression detection. miR-140 expression was detected using reverse transcription-quantitative polymerase chain reaction, and protein expression was measured by western blotting. TargetScan and dual luciferase reporter assays were used to reveal the association between miR-140 and programmed cell death-ligand 1 (PD-L1). BGC823 cell proliferation was detected by MTT assays. Ex vivo immune analysis by flow cytometry and ELISA were used to analyze immune function. It was demonstrated that miR-140 expression was significantly reduced in Hp-positive gastric cancer. PD-L1 was confirmed as a direct target of miR-140 in gastric cancer cells. In addition, PD-L1 expression was significantly increased in Hp-positive gastric cancer. Overexpression of miR-140 significantly suppressed gastric cancer cell proliferation through regulating PD-L1 expression. In vivo experiments also revealed that miR-140 markedly repressed tumor growth in the C57BL/6 mice. Furthermore, it was determined that the tumor-suppressive role of miR-140 in gastric cancer was associated with increased cytotoxic $\mathrm{CD} 8^{+} \mathrm{T}$ cell and reduced myeloid-derived suppressive and regulatory $\mathrm{T}$ cell infiltration. miR-140 significantly prevented mammalian target of
\end{abstract}

Correspondence to: Dr Jiangyang Lu, Department of Pathology, The First Affiliated Hospital of General Hospital of People's Liberation Army, 51 Fucheng Road, Haidian, Beijing 100048, P.R. China

E-mail: lujyhp2017@163.com

Key words: Helicobacter pylori, gastric cancer, microRNA-140, PD-L1/PD-1, proliferation rapamycin signaling in gastric cancer cells. Notably, these miR-140 overexpression-induced alterations were inhibited by PD-L1 plasmid. These findings indicated that miR-140 exerted an anti-gastric cancer effect by targeting immune checkpoint molecule PD-L1. Thus, miR-140 may be a promising and novel immunotherapeutic target for gastric cancer treatment.

\section{Introduction}

Gastric cancer, the fourth most common cancer in the world, is the second leading cause of cancer-associated mortality (1). The molecular mechanisms of gastric cancer have been extensively studied; however, the early diagnosis of gastric cancer remains uncommon (2), and the majority of patients have already reached an advanced stage at the time of diagnosis (3). The three major causes of gastric cancer include genetic predisposition, environmental factors and Helicobacter pylori (Hp) infection (4). Gastric carcinogenesis is a multi-step process that is closely associated with $\mathrm{Hp} \mathrm{(5).}$ Hp colonizes the stomach of $>50 \%$ of the world's population, and due to its pathogenic role in the development of gastric cancer, $\mathrm{Hp}$ is classified as a class I carcinogen (6). Therefore, there is an urgent need to identify novel bio-markers for the early diagnosis of gastric cancer, as well as new targets for gastric cancer therapy.

MicroRNAs (miRNAs/miRs) are a type of endogenous short non-coding RNA molecules 22 nucleotides in length, which directly bind to the 3'-untranslated region (3'-UTR) of multiple target mRNAs, promoting mRNA degradation and preventing translation to post-transcriptionally regulate gene expression. Numerous studies have indicated that abnormal expression of miRNAs is associated with the occurrence and progression of gastric cancer by regulating the expression of oncogenes and/or tumor inhibitor genes (7-12). In recent years, various studies of the role of miRNAs in gastric cancer, including Hp-positive gastric cancer, have been conducted. For example, it was reported that miR-101 functions as a growth-suppressive miRNA in Hp-associated gastric cancer by targeting suppressor of cytokine signaling 2 (13). miR-203 inhibits Hp-associated gastric cancer growth by repressing peripheral plasma membrane protein CASK expression (14). miR-Let-7c is significantly downregulated in Hp-positive gastric carcinogenesis (15). miR-24-3p regulates 
the progression of gastric mucosal lesions and suppresses the proliferation and invasiveness of N87 cells via peroxiredoxin 6 (16). Furthermore, miR-140, a well-studied tumor suppressor miRNA (17-19), has been found to inhibit gastric cancer growth via regulating YES proto-oncogene 1, Src family tyrosine kinase and transcription factor SRY-box 4 expression $(20,21)$. However, the role and mechanism of miR-140 in gastric cancer, particularly in the presence of $\mathrm{Hp}$, remains largely unclear.

Despite a number of achievements in chemotherapy and alternative therapeutic agents, there has been no major improvement in the overall survival in patients with gastric cancer over the past decade (22). Immunotherapy is a relatively recent strategy in gastric cancer therapy (23-25). However, the impact of immunotherapy in gastric cancer is unsatisfactory. Tumor cells escape T-cell-mediated cellular cytotoxicity through regulating the programmed cell death (PD)-1/PD-ligand 1 (PD-L1) pathway (26). Targeting the PD-1/PD-L1 pathway has emerged as a novel strategy in the treatment for a variety of malignancies (27-29). A previous study suggested that miR-140 exerts anti-osteosarcoma efficacy via targeting the immune checkpoint molecule PD-L1 (30). Therefore, the present study aimed to investigate the role and the molecular mechanism of miR-140 in Hp-associated gastric cancer, and to examine its association with immune function in gastric cancer.

\section{Materials and methods}

Clinical specimens. Gastritis tissue samples (15 Hp-positive, $15 \mathrm{Hp}$-negative) from 30 gastritis patients (21-53 years old; sex ratio: $1: 1)$, and 30 gastric cancer tissue samples $(15 \mathrm{Hp}$-positive and $15 \mathrm{Hp}$-negative) from 30 gastric cancer patients (with or without Hp infection; 23-57 years old; sex ratio: 1:1) were collected from patients who underwent gastroscopy at the hospital from December 2015 to December 2016. All the patients enrolled in the present study were $\geq 18$ years old, had no other cancer, and were not taking nonsteroidal anti-inflammatory drugs or proton pump inhibitors. In total, two gastric biopsies were collected from the patient: One biopsy was immediately frozen and stored at $-80^{\circ} \mathrm{C}$ until total RNA extraction, and the second biopsy was used for Hp detection. Hp infection was confirmed when a rapid urease test (31) was positive. The present study was approved by the Ethical Committee of the First Affiliated Hospital of General Hospital of People's Liberation Army, and written informed consent was obtained from each patient.

Cell culture. Gastric cancer cell lines AGS, MGC803 (cat. no. L4678; Wuhan Miaoling Bioscience \& Technology Co., Ltd., Wuhan, China), SGC7901(cat. no. L4801; Wuhan Miaoling Bioscience \& Technology Co., Ltd.), BGC823 (cat. no. L4448; Wuhan Miaoling Bioscience \& Technology Co., Ltd.) and MKN45 (cat. no. L4679; Wuhan Miaoling Bioscience \& Technology Co., Ltd.), and the immortalized non-tumorigenic cell line GES-1 were purchased from American Type Culture Collection (Manassas, VA, USA). Cells were cultured in Dulbecco's modified Eagle's medium or RPMI-1640 (both Gibco; Thermo Fisher Scientific, Inc., Waltham, MA USA) containing $10 \%$ heat-inactivated fetal bovine serum (Gibco; Thermo Fisher Scientific, Inc.) and 1\% streptomycin-penicillin solution (Sigma-Aldrich; Merck KGaA, Darmstadt, Germany) at $37^{\circ} \mathrm{C}$ with $5 \% \mathrm{CO}_{2}$.

BGC823 cells were infected with different multiplicity of infections (MOIs) of $\mathrm{Hp}(0,1: 1,1: 50$ or 1:100; cat. no. MA135390; Thermo Fisher Scientific, Inc.,).

Cell transfection. miR-140 mimic (5'-UGAGAACUGAAU UCCAUGGGUU-3') and mimic control (5'-UUCUCCGAA CGUGUCACGUTT-3') were obtained from Sangon Biotech Co., Ltd. (Shanghai, China). For cell transfection, BGC823 cells were seeded in 6 -well plates $\left(4 \times 10^{5}\right.$ per well). Then $100 \mathrm{nM}$ miR-140 mimic, $100 \mathrm{nM}$ mimic control, $100 \mathrm{nM}$ miR-140 mimic + $1 \mu \mathrm{g}$ control-plasmid (cat. no. sc-108083; Santa Cruz Biotechnology, Inc., Dallas, TX, USA), or $100 \mathrm{nM}$ miR-140 mimic + $1 \mu \mathrm{g}$ PD-L1-plasmid (cat. no. sc-401140-ACT; Santa Cruz Biotechnology, Inc.) was transfected into BGC823 cells using Lipofectamine ${ }^{\circledR} 3000$ (Invitrogen; Thermo Fisher Scientific, Inc.) according to the manufacturer's protocol. Cells were subjected to further experimentation $24 \mathrm{~h}$ after transfection. Transfection efficiency was detected by reverse transcription-quantitative polymerase chain reaction (RT-qPCR).

MTT assay. BGC823 cells ( $5 \times 10^{3}$ cells/well) were cultured in 96-well plates. Cells were transfected with miR-140 mimic, mimic control, miR-140 mimic + control - plasmid, or miR-140 mimic + PD-L1 - plasmid at $37^{\circ} \mathrm{C}$ for $24 \mathrm{~h}$; following this, MTT solution $(20 \mu \mathrm{l})$ was added into each well at 24,48 and $72 \mathrm{~h}$, and plates were then incubated at $37^{\circ} \mathrm{C}$ for another 4 h. Then DMSO (100 $\mu$; Nanjing KeyGen Biotech. Co. Ltd., Nanjing, China) was added to dissolve the formazan crystals. To determine cell viability, the absorbance was measured at $450 \mathrm{~nm}$.

$R T$ - $q P C R$. Total RNA from tissues and cells was isolated using a RNeasy Mini kit (Qiagen, Inc., Valencia, CA, USA) following the manufacturer's protocol. RT was performed to synthesize cDNA using the TaqMan MicroRNA Reverse Transcription kit (Applied Biosystems; Thermo Fisher Scientific, Inc.) according to the manufacturer's protocol. Reaction conditions for RT were: $50^{\circ} \mathrm{C}$ for $15 \mathrm{~min}$ and $85^{\circ} \mathrm{C}$ for 2 min. SYBR Premix Ex Taq ${ }^{\mathrm{TM}}$ II (TliRNaseH Plus) kit (Takara Bio, Inc., Otsu, Japan) was used to perform qPCR. Amplification conditions for qPCR were as follows: $95^{\circ} \mathrm{C}$ for $5 \mathrm{~min}$, followed by 40 cycles of denaturation at $95^{\circ} \mathrm{C}$ for $15 \mathrm{sec}$ and annealing/elongation at $60^{\circ} \mathrm{C}$ for $30 \mathrm{sec}$. U6 or GAPDH was used as internal control. The primer sequences used were listed in Table I. Relative gene quantification was assessed using the $2^{-\Delta \Delta \mathrm{Cq}}$ method (32).

Western blot analysis. Proteins were extracted from cells or tissues with radioimmunoprecipitation assay lysis buffer (Beyotime Institute of Biotechnology, Haimen, China). A BCA protein assay kit (Pierce; Thermo Fisher Scientific, Inc.) was used to evaluate the protein concentrations. Protein samples ( $25 \mu \mathrm{g} /$ lane) were separated by $10 \%$ SDS-PAGE, and then transferred to polyvinylidene difluoride membranes. Membranes were blocked with $5 \%$ non-fat milk for $2 \mathrm{~h}$ at room temperature, and then incubated with primary antibodies against PD-L1 (cat. no. 13684; 1:1,000; Cell Signaling Technology, Inc., 
Table I. Primer sequences used for quantitative polymerase chain reaction analysis.

\begin{tabular}{lcl}
\hline Primer & Direction & \multicolumn{1}{c}{ Sequence (5'-3') } \\
\hline PD-L1 & $\mathrm{F}$ & GGCATTTGCTGAACGCAT \\
& $\mathrm{R}$ & CAATTAGTGCAGCCAGGT \\
IFN- $\gamma$ & $\mathrm{F}$ & CTAATTATTCGGTAACTGACTTGA \\
& $\mathrm{R}$ & ACAGTTCAGCCATCACTTGGA \\
TNF- $\alpha$ & $\mathrm{F}$ & CCTCTTCTCATTCCTGCTC \\
& $\mathrm{R}$ & CTTCTCCTCCTTG TTGGG \\
miR-140 & $\mathrm{F}$ & CGCGCCAGTGGTTTACCCT \\
& $\mathrm{R}$ & CCAGTGCAGGGTCCGAGGTA \\
U6 & $\mathrm{F}$ & GGAGCGAGATCCCTCCAAAAT \\
& $\mathrm{R}$ & GGCTGTTGTCATACTTCTCATGG \\
GAPDH & $\mathrm{F}$ & CTTTGGTATCGTGGAAGGACTC \\
& $\mathrm{R}$ & GTAGAGGCAGGGATGATGTTCT \\
\hline
\end{tabular}

F, forward; R, reverse; IFN, interferon; TNF, tumor necrosis factor; miR, microRNA; PD-L1, programmed cell death ligand 1.

Danvers, MA, USA), phosphorylated (p)-mammalian target of rapamycin (mTOR; cat. no. 5536; 1:1,000; Cell Signaling Technology, Inc.), p-ribosomal protein s6 kinase $\beta$-1 (S6K1; cat. no. ab60948; 1:1,000; Abcam, Cambridge, MA, USA) and $\beta$-actin (cat no. 4970; 1:2,000; Cell Signaling Technology, Inc.) at $4^{\circ} \mathrm{C}$ overnight. Subsequently, the PVDF membranes were incubated with horseradish peroxidase-conjugated anti-rabbit IgG secondary antibody (cat no. 7074; dilution ratio: 1:5,000; Cell Signaling Technology, Inc.) at room temperature for $1.5 \mathrm{~h}$. Protein blots were visualized using the SuperSignal West Femto Maximum Sensitivity Substrate (Pierce; Thermo Fisher Scientific, Inc.) according to the manufacturer's protocol.

Luciferase reporter assay. TargetScan 7.1 (www.targetscan. org/vert_71) was used to predict the target genes of miR-140, and PD-L1 was identified as a potential target of miR-140. To confirm direct target binding, the wild type (WT) and mutant (MUT) 3'-UTR of PD-L1 was cloned into a pmiR RB Report ${ }^{\mathrm{TM}}$ dual luciferase reporter gene plasmid vector (Guangzhou RiboBio Co., Ltd., Guangzhou, China). BGC823 cells were co-transfected with $100 \mathrm{ng}$ WT-PD-L1 or $100 \mathrm{ng}$ MUT-PD-L1 and $50 \mathrm{nM}$ miR-140 mimic or its control $(50 \mathrm{nM}$; miR-C) vector using Lipofectamine ${ }^{\circledR} 3000$, according to the manufacturer's protocols. After $48 \mathrm{~h}$, luciferase activity was measured by the dual-luciferase assay system (Promega Corporation, Madison, WI, USA) as per the manufacturer's protocol, and normalized to Renilla luciferase activity.

Animal experiments. A total of 50 male C57BL/6 mice (8 weeks old; $22 \mathrm{~g}$ ) were purchased from the Laboratory Animal Center of the Academy of Military Medical Sciences (Beijing, China). Mice had free access to food and water. All mice were fed ad libitum and maintained under standard conditions at $22-30^{\circ} \mathrm{C}$ and a $12-\mathrm{h} \mathrm{light} / \mathrm{dark}$ cycle. The experimental protocol was approved by the Ethical Committee of the First Affiliated
Hospital of General Hospital of People's Liberation Army, and all experiments were applied according to the guidance of the Laboratory Animal Care (NIH publication no. 85Y23, revised 1996) (33). For generation of subcutaneous tumors, BGC823 cells $\left(5 \times 10^{6}\right)$ were injected subcutaneously into the flanks of mice. When the tumor size reached approximately $50 \mathrm{~mm}^{3}$, the mice were divided into five groups: Control, miR-140 mimic (mice were injected with $50 \mu 1$ solution containing $7 \mathrm{nmol} \mathrm{miR-140} \mathrm{mimic,} 3 \mu$ l Lipofectamine ${ }^{\circledR} 2000$ (Invitrogen; Thermo Fisher Scientific, Inc.), and $40 \mu 1$ of serum-free medium Opti-MEM (Invitrogen), mimic control [mice were injected with $50 \mu$ l solution containing $7 \mathrm{nmol} \mathrm{mimic} \mathrm{control,}$ $3 \mu 1$ Lipofectamine ${ }^{\circledR} 2000$ (Invitrogen; Thermo Fisher Scientific, Inc.), and $40 \mu 1$ of serum-free medium Opti-MEM (Invitrogen; Thermo Fisher Scientific, Inc.)], miR-140 mimic + control-plasmid [mice were injected with $50 \mu 1$ solution containing $7 \mathrm{nmol} \mathrm{miR-140} \mathrm{mimic,} 1 \mu \mathrm{g}$ control-plasmid, $3 \mu 1$ Lipofectamine ${ }^{\circledR} 2000$ (Invitrogen; Thermo Fisher Scientific, Inc.), and $40 \mu 1$ of serum-free medium Opti-MEM (Invitrogen; Thermo Fisher Scientific, Inc.)], and miR-140 mimic + PD-L1-plasmid [mice were injected with $50 \mu 1$ solution containing $7 \mathrm{nmol} \mathrm{miR-140} \mathrm{mimic,} 1 \mu \mathrm{g}$ PD-L1-plasmid, $3 \mu 1$ Lipofectamine ${ }^{\circledR} 2000$ (Invitrogen; Thermo Fisher Scientific, Inc.), and $40 \mu \mathrm{l}$ of serum-free medium Opti-MEM (Invitrogen; Thermo Fisher Scientific, Inc.)]. Injections were performed once a day for 19 days. Tumors were measured every week. Tumor volume was calculated using the following formula: Volume $=($ length $) \times(\text { width })^{2} / 2$.

Ex vivo immune analysis. After 19 days, mice were sacrificed and tumors were dissected and weighed. Single-cell suspensions were extracted from the tumors. Briefly, the tumor tissues were cut into small pieces $(1-2 \mathrm{~mm})$, washed three times with PBS buffer, and transferred to a $50 \mathrm{ml}$ centrifuge tube. Depending on the amount of tissue, 5-6 times of $0.25 \%$ trypsin solution was added and the tissues digested at $37^{\circ} \mathrm{C}$ for 20-40 min. After standing for 2-3 min, the suspension was transferred to a new centrifuge tube. The suspension was filtered twice with a 200/300 mesh nylon mesh. The filtered suspension was centrifuged at $1,200 \mathrm{x}$ g for $10 \mathrm{~min}$ at $4^{\circ} \mathrm{C}$ and the supernatant was discarded. Then, $5 \mathrm{ml}$ of PBS buffer was added, the cells gently dispersed, and centrifuged again $\left(1,200 \mathrm{x} \mathrm{g}\right.$ for $10 \mathrm{~min}$ at $\left.4^{\circ} \mathrm{C}\right) . \mathrm{CD} 8^{+} \mathrm{T}$ cells, $\mathrm{T}$ regulatory cells (Tregs), and myeloid-derived suppressor cells (MDSCs) in the tumor cell suspension were identified by flow cytometry. Data were analyzed using FlowJo software v7.6 (FlowJo LLC, Ashland, OR, USA).

ELISA. The serum level of IL-10 was measured using the ELISA kit (cat no. ab108870; Abcam, Cambridge, MA, USA) in accordance with the manufacturer's instructions.

Statistical analysis. All the experiments were performed three times. Statistical analysis was performed with SPSS software version 20.0 (IBM Corp., Armonk, NY, USA). Data were displayed as the mean \pm standard deviation of three experimental repeats. Statistical comparisons between groups were made by one-way analysis of variance with Tukey's post-hoc test, or Student's t-test. $\mathrm{P}<0.05$ was considered to indicate a statistically significant difference. 
A

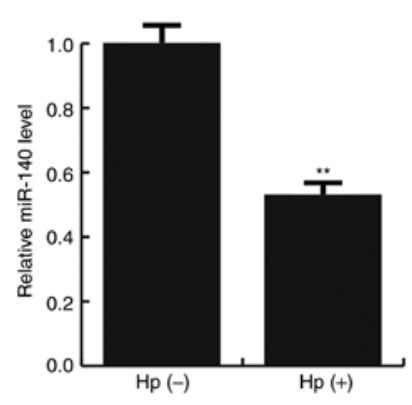

C

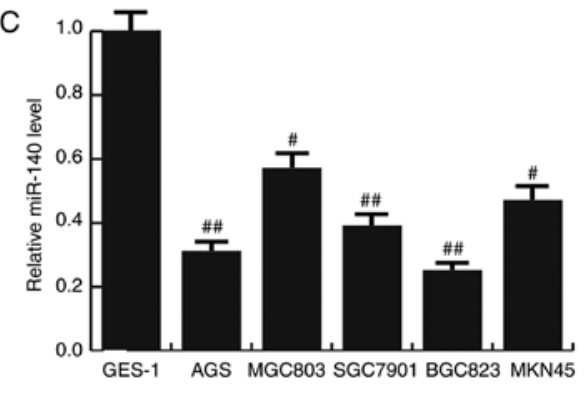

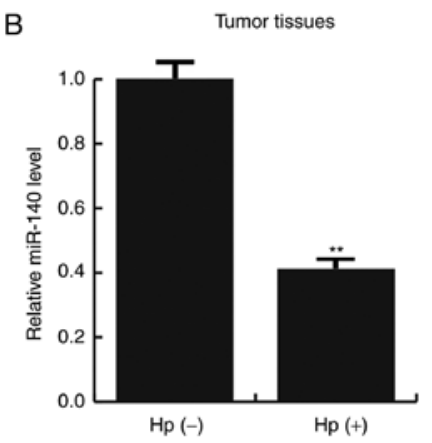

D

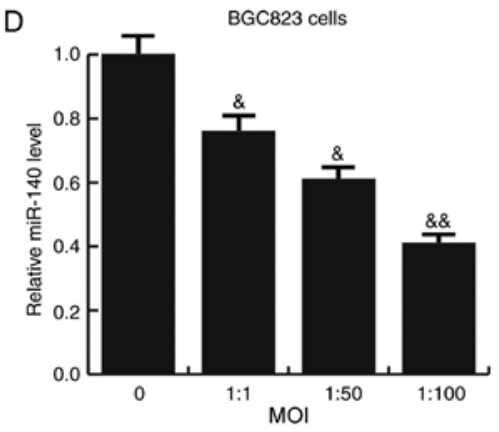

Figure 1. miR-140 expression is downregulated in Hp-positive gastric cancer tissues and cell lines. (A) miR-140 expression in Hp-positive and -negative normal control tissues, as well as (B) Hp-positive and -negative gastric cancer tissues. ${ }^{* *} \mathrm{P}<0.01$ vs Hp-negative group. (C) miR-140 expression in five gastric cancer cell lines and immortalized GES-1 cells. ${ }^{~} \mathrm{P}<0.05,{ }^{\# \#} \mathrm{P}<0.01$ vs. GES-1 cells. (D) miR-140 expression in BGC823 cells infected with different MOIs of Hp. ${ }^{\circledR} \mathrm{P}<0.05$, ${ }^{\&} \mathrm{P}<0.01$ vs. 0 group. Data are presented as the mean \pm standard deviation of three independent experiments. miR, microRNA; Hp, Helicobacter pylori; MOI, multiplicity of infection.

\section{Results}

miR-140 is aberrantly downregulated in Hp-positive tissues and cells. The expression levels of miR-140 in Hp-positive and Hp-negative gastric cancer tissues, as well as Hp-positive and Hp-negative normal tissues was detected using RT-qPCR. It was revealed that the average expression level of miR-140 was significantly lower in Hp-positive tumor and normal tissues (Fig. 1A and B). Next, the expression of miR-140 in various human gastric cancer cell lines (AGS, MGC803, SGC7901, BGC823 and MKN45) was examined. Consistent with the tissue results, compared with the immortalized non-tumorigenic cell line GES-1, miR-140 was significantly decreased in all 5 GC cell lines examined (Fig. 1C). BGC823 cells had the lowest miR-140 expression, and were therefore selected for further functional analysis. BGC823 cells were infected with different MOIs of $\mathrm{Hp}(0,1: 1,1: 50,1: 100)$. The results indicated as the MOI increased, miR-140 expression gradually reduced (Fig. 1D). These results indicated that miR-140 expression was significantly downregulated in $\mathrm{Hp}$-infected conditions and may be associated with gastric cancer progression.

PD-L1 is directly targeted by miR-140 in gastric cancer. TargetScan was used to predict the potential targets of miR-140, and PD-L1 was identified as a potential target of miR-140 (Fig. 2A). To investigate whether PD-L1 was a direct target of miR-140, a luciferase reporter assay was constructed. The results indicated that miR-140 significantly reduced the luciferase activity of PD-L1-WT in BGC823 cells, but had no effect on the mutant form of PD-L1-MUT (Fig. 2B), indicating that PD-L1 was directly targeted by miR-140 in gastric cancer cells.
A PD-L1 3'-UTR

WT 3' UTR 5' -UCCAGCAUUGGAACUUCUGAUCu 3' | | | | | | | | | | | | miR-140 $3^{\prime}$ ucGGUGUUAG UGGAAGACUAGa $5^{\prime}$ Mut 3' UTR 5' -UCCAGCAUUGGAACGGCACGUCu 3'

B

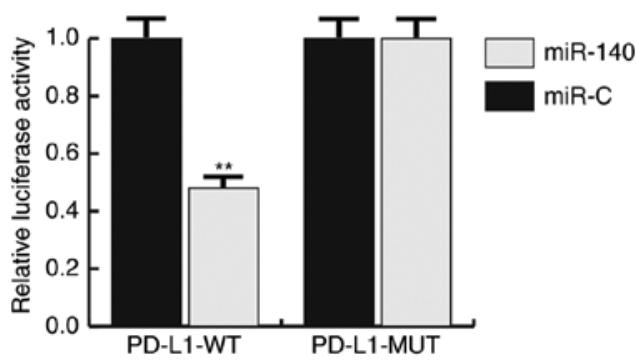

Figure 2. PD-L1 is a direct target of miR-140. (A) The interaction between miR-140 and the 3'-UTR of PD-L1 was predicted using TargetScan. (B) Luciferase activity of a reporter containing a PD-L1-WT 3'-UTR or PD-L1-MUT 3'-UTR. ${ }^{* *} \mathrm{P}<0.01$ vs miR-C. Data are presented as the mean \pm standard deviation of three independent experiments UTR, untranslated region; PD-L1, programmed cell death ligand 1; MUT, mutant; WT, wild type; miR, microRNA; miR-C, miR-140 mimic control.

PD-L1 is upregulated in Hp-positive tumor cells and tissues. The expression level of PD-L1 in Hp-positive and -negative gastric cancer tissues and cells was subsequently determined. It was found that the mRNA expression of PD-L1 was significantly higher in Hp-positive normal (Fig. 3A) and tumor tissues (Fig. 3B). PD-L1 expression was also detected in five human gastric cancer cell lines, and the results revealed that compared with GES-1 cells, PD-L1 mRNA (Fig. 3C) and protein (Fig. 3D) expression was significantly increased in all the gastric cancer cells. In addition, as cells were transfected 
A

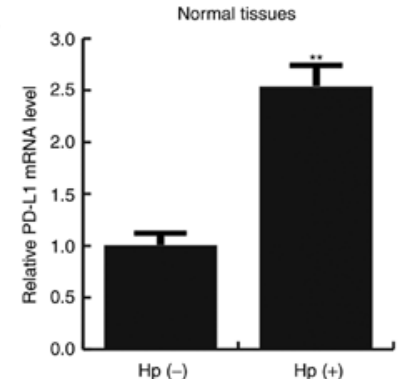

B

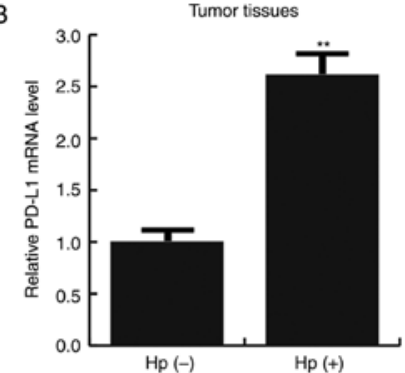

C

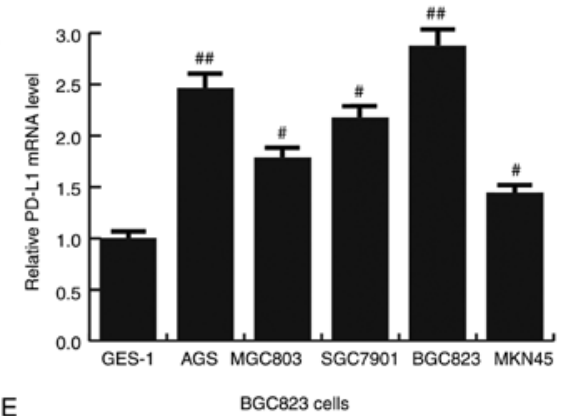

D

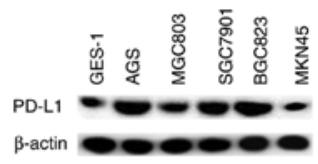

E

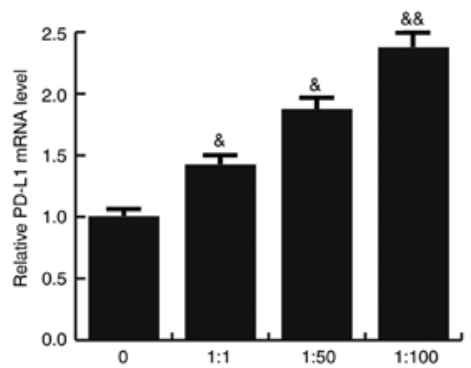

F

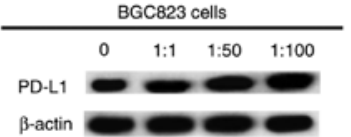

Figure 3. PD-L1 expression in upregulated in Hp-positive gastric cancer tissues and cell lines. (A) PD-L1 mRNA expression in Hp-positive and negative normal tissues, as well as (B) Hp-positive and negative gastric cancer tissues. ${ }^{* *} \mathrm{P}<0.01$ vs Hp-negative group. (C) PD-L1 mRNA and (D) protein expression in give gastric cancer cell lines and immortalized GES-1 cells. ${ }^{\#} \mathrm{P}<0.05,{ }^{\# \#} \mathrm{P}<0.01$ vs. GES-1 cells. (E) PD-L1 mRNA and (F) protein expression in BGC823 cells infected with different MOIs of $\mathrm{Hp}(0,1: 1,1: 50,1: 100) .{ }^{~} \mathrm{P}<0.05$, \&\& $\mathrm{P}<0.01$ vs. 0 MOI group. Data are presented as the mean \pm standard deviation of three independent experiments. miR, microRNA; PD-L1, programmed cell death ligand 1; Hp, Helicobacter pylori; MOI, multiplicity of infection.
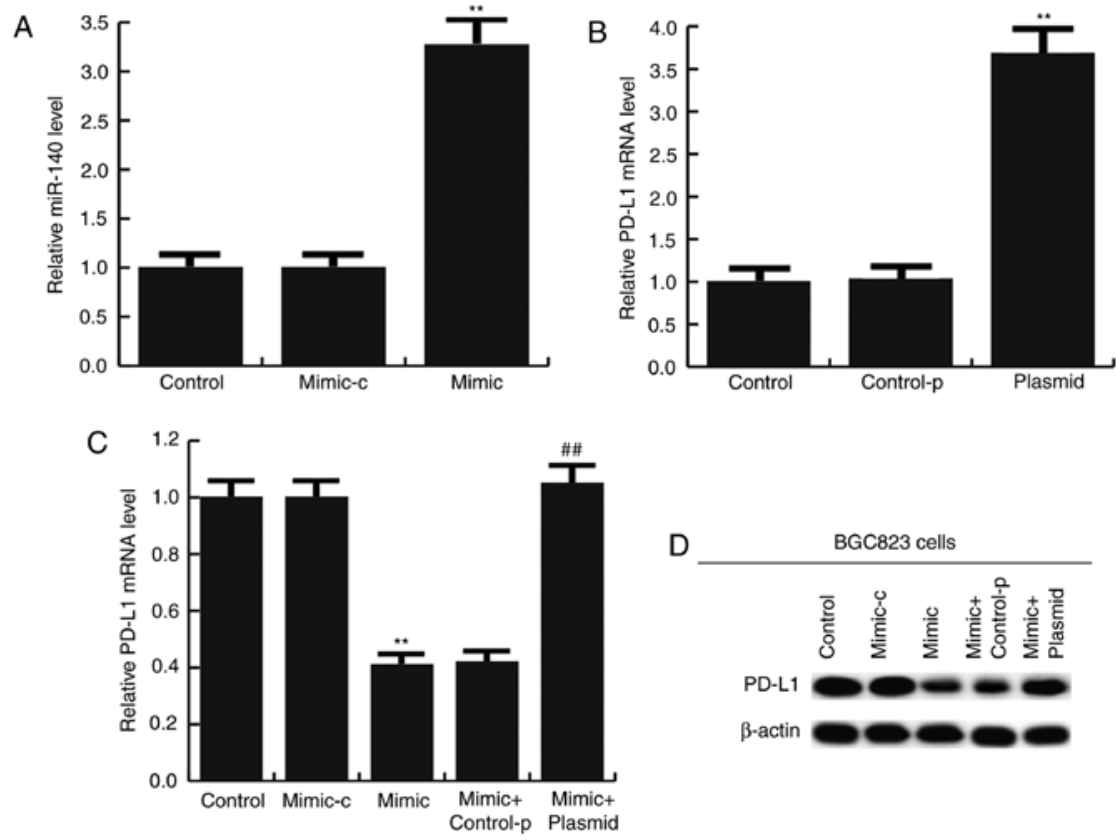

D BGC823 cells

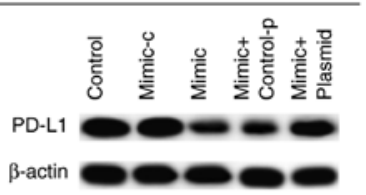

Figure 4. miR-140 mimic suppresses PD-L1 expression in BGC823 cells. Following transfection with miR-140 mimic, mimic-c, miR-140 mimic + control-p or miR-140 mimic + PD-L1-plasmid for $24 \mathrm{~h}$, the expression of miR-140 and PD-L1 in BGC823 cells was detected. (A) miR-140 mimic significantly enhanced miR-140 expression in BGC823 cells, and (B) PD-L1-plasmid significantly enhanced PD-L1 mRNA expression in BGC823 cells. (C) PD-L1 mRNA and (D) protein expression was examined in each group. Data are presented as the mean \pm standard deviation of three independent experiments. ${ }^{* *} \mathrm{P}<0.01 \mathrm{vs}$. control; ${ }^{\# \#} \mathrm{P}<0.01$ vs. mimic. miR, microRNA; PD-L1, programmed cell death ligand 1; mimic-c, miR-140 mimic control; control-p, control plasmid. 

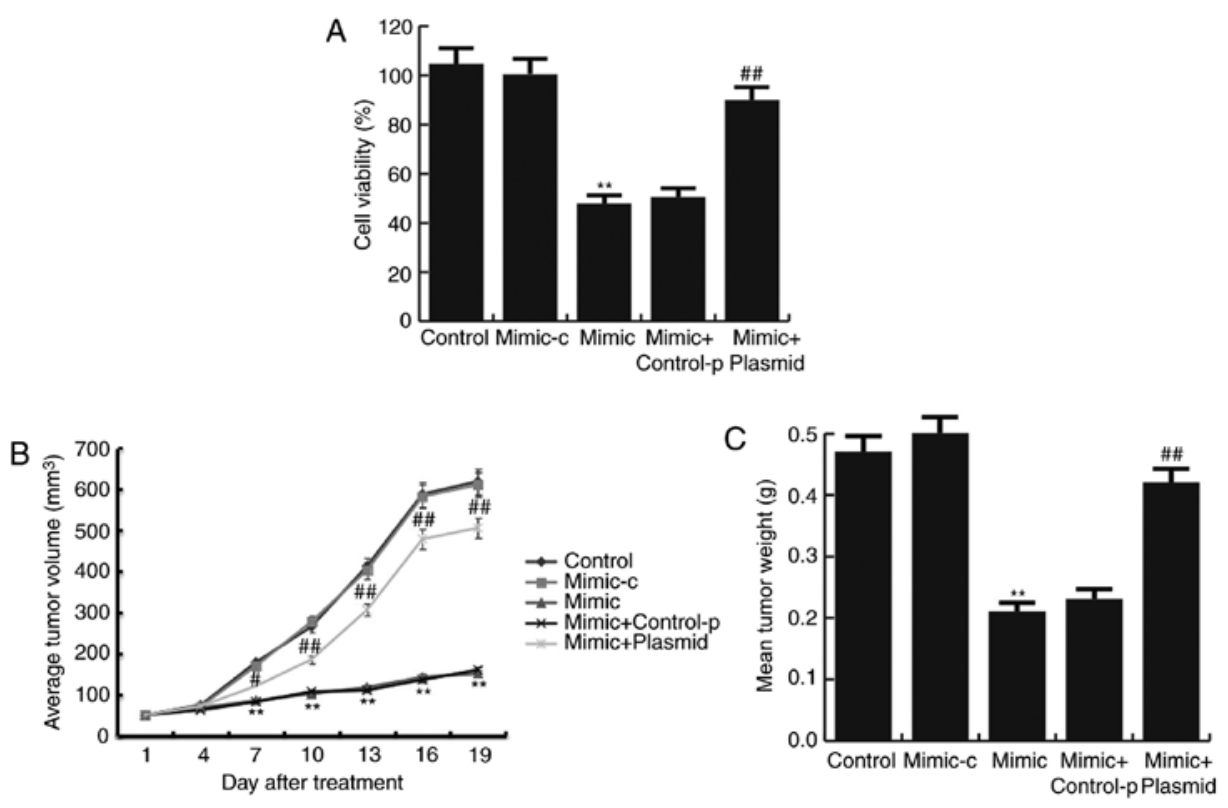

Figure 5. miR-140 mimic reduces gastric cancer cell viability. (A) Following transfection with miR-140 mimic, mimic-c, miR-140 mimic + control-p, or miR-140 mimic + PD-L1-plasmid for $24 \mathrm{~h}$, BGC823 cell viability was assessed with MTT assays. (B) Tumor size at different time points following the intratumoral injection of miR-140 mimic, mimic control, miR-140 mimic + control-p, or miR-140 mimic + PD-L1-plasmid. (C) Mean weights of the xenograft tumors. Data are presented as the mean \pm standard deviation of three independent experiments. ${ }^{* *} \mathrm{P}<0.01$ vs. control; ${ }^{\#} \mathrm{P}<0.05$, ${ }^{\# \#} \mathrm{P}<0.01$ vs. mimic. miR, microRNA; PD-L1, programmed cell death ligand 1; mimic-c, miR-140 mimic control; control-p, control plasmid.

with a higher MOI of Hp, PD-L1 mRNA (Fig. 3E) and protein (Fig. 3F) expression was gradually increased.

miR-140 has a tumor-suppressive role in gastric cancer. To determine the potential role of miR-140 in gastric cancer, BGC823 cells were transfected with miR-140 mimic, mimic-c, miR-140 mimic + control-plasmid, or miR-140 mimic + PD-L1-plasmid for $24 \mathrm{~h}$. Transfection efficiency was initially measured using RT-qPCR. The results confirmed that compared with the control group, miR-140 expression was significantly increased in the miR-140 mimic group (Fig. 4A), and PD-L1-plasmid significantly enhanced PD-L1 expression (Fig. 4B). mRNA (Fig. 4C) and protein (Fig. 4D) expression of PD-L1 was detected and the results indicated that miR-140 mimic notably reduced PD-L1 expression, and this reduction was inhibited by PD-L1-plasmid co-transfection (Fig. 4C and D).

Next, the proliferation of BGC823 cells was detected by a MTT assay. It was demonstrated that compared with the control group, miR-140 mimic significantly inhibited BGC823 cell proliferation, and this inhibition was prevented by PD-L1-plasmid co-transfection (Fig. 5A). Consistent with the data obtained from in vitro experiments, results from the in vivo experiments showed that tumor volume (Fig. 5B) and mean tumor weight (Fig. 5C) was significantly reduced in the miR-140 mimic group, compared with the control group, and PD-L1-plasmid co-transfection significantly increased these parameters.

miR-140 enhances antitumor immunity in gastric cancer. Studies have indicated that PD-L1/PD-1 signaling inhibition may prevent immune suppression and enhance antitumor response (26). Thus, it was investigated whether miR-140 affected the immune response in gastric cancer. As presented in Fig. 6A, compared with the control group, the $\mathrm{CD} 8^{+} \mathrm{T}$ cell population in the miR-140 mimic group was significantly increased, whereas the MDSC and Tregs cell populations were significantly reduced. These alterations were prevented by PD-L1 overexpression. To further characterize the immune responses induced by miR-140, the mRNA expression of IFN- $\gamma$ and TNF- $\alpha$ was measured in tumor tissues, as well as serum IL-10 expression. As expected, miR-140 mimic significantly increased IFN- $\gamma$ (Fig. 6B) and TNF- $\alpha$ (Fig. 6C) mRNA expression and decreased serum IL-10 expression (Fig. 6D), compared with the control group. These alterations were eliminated by PD-L1 overexpression.

miR-140 suppresses mTOR signaling in gastric cancer. Previous studies have reported that cell-intrinsic PD-L1/PD-1 signaling promotes tumor growth by activating downstream mTOR signaling (34). Therefore, the effects of miR-140 on mTOR signaling in gastric cancer were examined. As presented in Fig. 7, compared with the control group, p-mTOR and $\mathrm{p}-\mathrm{S} 6 \mathrm{~K} 1$ expression was significantly decreased, and PD-L1 overexpression prevented this decrease. These data indicated that miR-140 suppressed mTOR signaling in gastric cancer.

\section{Discussion}

To the best of our knowledge, the present study was the first to identify that miR-140 directly regulated PD-L1 expression in gastric cancer. Upregulation of miR-140 inhibited gastric cancer growth by targeting PD-L1 in vitro and in vivo, and was associated with an enhanced antitumor immunity. Therefore, miR-140 may represent a novel and promising therapeutic target for gastric cancer treatment through immune checkpoint inhibition. 

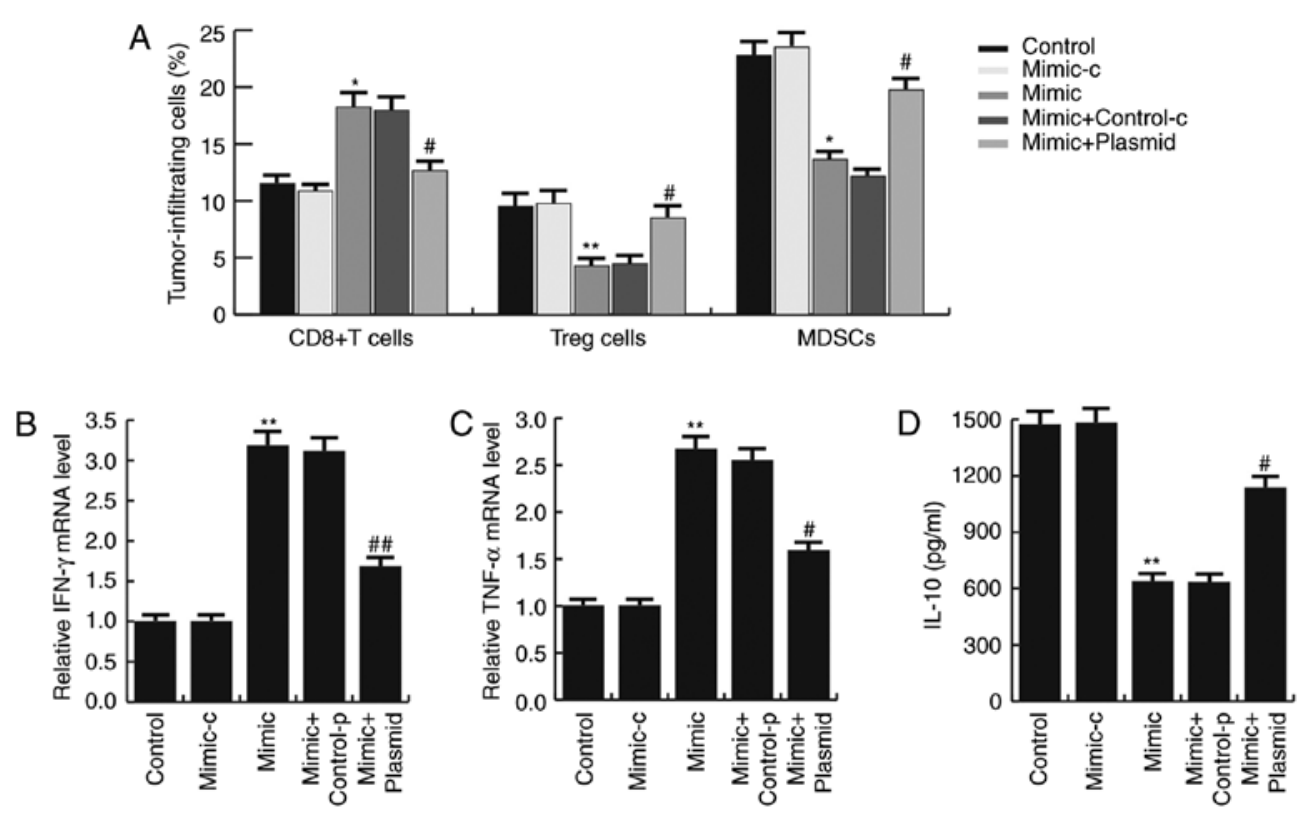

Figure 6. miR-140 enhances antitumor immunity in gastric cancer. (A) Ex vivo analysis of the CD8 ${ }^{+} \mathrm{T}$ cell, Treg cell and MDSC populations in the tumors of each group. Cells were isolated and analyzed by flow cytometry. (B) Relative mRNA expression of IFN- $\gamma$ and (C) TNF- $\alpha$. (D) Serum IL-10 expression in each group was analyzed by ELISA. Data are presented as the mean \pm standard deviation of three independent experiments. ${ }^{*} \mathrm{P}<0.05$, ${ }^{* *} \mathrm{P}<0.01$ vs. control. ${ }^{\#} \mathrm{P}<0.05$, ${ }^{\# \#} \mathrm{P}<0.01$ vs. mimic. miR, microRNA; Treg, T regulatory; MDSC, myeloid-derived suppressor cell; IFN- $\gamma$, interferon $\gamma$; TNF- $\alpha$, tumor necrosis factor- $\alpha$; IL-10, interleukin-10; mimic-c, miR-140 mimic control; control-p, control plasmid.

In recent years, miRNA-based immunotherapies have emerged as novel treatments for malignant tumors (35). Tumor cells may escape T-cell-mediated cellular cytotoxicity by exploiting the PD-1/PD-L1 pathway (26). PD-1 and PD-L1, once bound, transmit negative regulatory signals to $\mathrm{T}$ cells to induce a resting state, which decreases lymph node $\mathrm{CD}^{+} \mathrm{T}$ cell proliferation and cancer cell recognition. In addition, $T$ cell apoptosis is induced to effectively reduce the body's immune response, leading to the unrestrained growth of cancer cells. Therefore, targeting the PD-1/PD-L1 pathway is a promising strategy for the treatment of malignant tumors. Evidence has indicated that miRNAs regulate PD-L1 expression in cancer (36). For example, downregulation of miR-140-3p is closely associated with overexpression of PDL-1 in many cancers, including breast and lung cancer (37). In malignant pleural mesothelioma, tumor suppressor gene miRNAs (miR-15b, miR-16, miR-193a-3p, miR-195, and miR-200c) downregulate the expression of PD-L1 (38). In pancreatic cancer, miR-142-5p can inhibit PD-L1 expression and enhance antitumor immunity (39). The miR-25-93-106b cluster has been reported to regulate tumor metastasis and immune evasion by regulating stromal cell-derived factor 1 and PD-L1 expression (40). miR-424 reduces ovarian cancer chemoresistance by inhibiting PD-L1 expression (41). miR-140, a well-studied miRNA in cancer, has been identified as a tumor suppressor in various cancer types, including gastric cancer (17-21). In addition, miR-140 has been demonstrated to suppress osteosarcoma tumor growth by enhancing antitumor immune response, by regulating PD-L1 (30). However, the role and mechanism of miR-140 in gastric cancer, particularly in Hp-associated gastric cancer, remained largely unclear. Therefore, the present study was conducted.

First, the present study detected the expression of miR-140 in Hp-positive and -negative gastric cancer tissues,

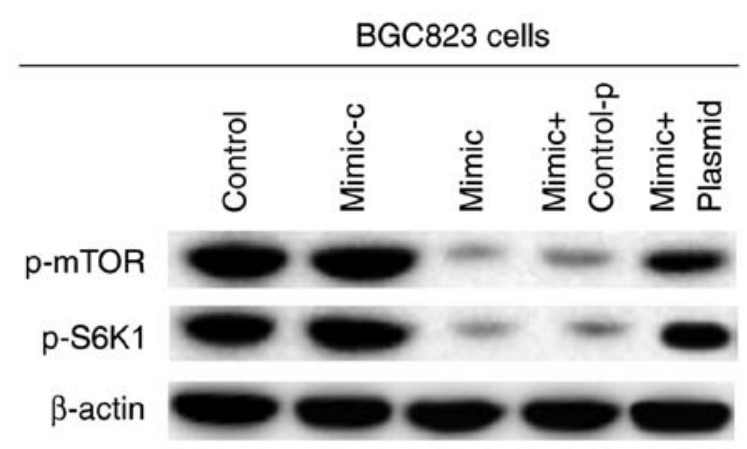

Figure 7. miR-140 inhibits mTOR signaling in gastric cancer. Following transfection with miR-140 mimic, mimic-c, miR-140 mimic + control-p or miR-140 mimic + PD-L1-plasmid for $24 \mathrm{~h}$, the protein expression of p-mTOR and p-S6K1 in BGC823 cells was measured by western blotting. miR, microRNA; p-, phosphorylated; mTOR, mammalian target of rapamycin; S6K1, ribosomal protein s6 kinase $\beta-1$; mimic-c, miR-140 mimic control; control-p, control plasmid.

Hp-positive and -negative normal tissues, and five human gastric cancer cell lines. The results indicated that miR-140 expression was significantly downregulated in Hp-positive conditions and was associated with gastric cancer progression. It was then confirmed that PD-L1 was a direct target of miR-140 in gastric cancer cells, and was upregulated in Hp-positive tumor cells and tissues. Further analysis indicated that miR-140 suppressed gastric cancer growth in vitro and in vivo, and enhanced antitumor immunity in gastric cancer. Previous studies have reported that cell-intrinsic PD-L1/PD-1 signaling promotes tumor growth by activating downstream mTOR signaling (34). Consistent with the results of previous research $(30,34)$, the present study demonstrated that miR-140 suppressed mTOR signaling in gastric cancer. 
Taken together, the current study revealed that miR-140 was significantly reduced in Hp-positive gastric cancer, and exerted a tumor suppressive effect by targeting immune checkpoint molecule PD-L1. Thus, miR-140 may be a novel and promising therapeutic target for the treatment of gastric cancer, particularly in Hp-positive gastric cancer.

\section{Acknowledgements}

Not applicable.

\section{Funding}

The present study was supported by the Fund of Development of Health Service of Beijing (grant no. 2011-5002-02).

\section{Availability of data and materials}

The datasets used and/or analyzed during the current study are available from the corresponding author on reasonable request.

\section{Authors' contributions}

MZ performed study design, data collection, statistical analysis, data interpretation, manuscript preparation and literature search. QL performed study design, statistical analysis and data interpretation. WL performed data collection. $\mathrm{HZ}$ performed study design, data collection and statistical analysis. XZ performed data collection and statistical analysis. JL performed study design, data interpretation and manuscript preparation.

\section{Ethics approval and consent to participate}

The present study was approved by the Ethical Committee of the First Affiliated Hospital of General Hospital of People's Liberation Army, and written informed consent was obtained from each patient.

\section{Patient consent for publication}

Not applicable.

\section{Competing interests}

The authors declare that they have no competing interests.

\section{References}

1. Muhammad JS, Sugiyama T and Zaidi SF: Gastric pathophysiological ins and outs of helicobacter pylori: A review. J Pak Med Assoc 63: 1528-1533, 2013

2. Ali Z, Deng Y and Ma C: Progress of research in gastric cancer. J Nanosci Nanotechnol 12: 8241-8248, 2012.

3. Lordick F, Allum W, Carneiro F, Mitry E, Tabernero J, Tan P, Van Cutsem E, van de Velde C and Cervantes A: Unmet needs and challenges in gastric cancer: The way forward. Cancer Treat Rev 40: 692-700, 2014.

4. Graham DY: History of Helicobacter pylori, duodenal ulcer, gastric ulcer and gastric cancer. World J Gastroenterol 20: 5191-5204, 2014.

5. Falt P, Hanousek M, Kundrátová E and Urban O: Precancerous conditions and lesions of the stomach. Klin Onkol 26 (Suppl): S22-S28, 2013 (In Czech).
6. Xiao D, Zhang H, He L, Peng X, Wang Y, Xue G, Su P and Zhang J: High natural variability bacteria identification and typing: Helicobacter pylori analysis based on peptide mass fingerprinting. J Proteomics 98: 112-122, 2014.

7. Katada T, Ishiguro H, Kuwabara Y, Kimura M, Mitui A, Mori Y, Ogawa R, Harata K and Fujii Y: MicroRNA expression profile in undifferentiated gastric cancer. Int J Oncol 34: 537-542, 2009.

8. Bou Kheir T, Futoma-Kazmierczak E, Jacobsen A, Krogh A Bardram L, Hother C, Grønbæk K, Federspiel B, Lund AH and Friis-Hansen L: miR-449 inhibits cell proliferation and is down-regulated in gastric cancer. Mol Cancer 10: 29, 2011.

9. Chiang Y, Zhou X, Wang Z, Song Y, Liu Z, Zhao F, Zhu J and $\mathrm{Xu} \mathrm{H}$ : Expression levels of microRNA-192 and -215 in gastric carcinoma. Pathol Oncol Res 18: 585-591, 2012.

10. Zhang H, Cheng Y,Jia C, Yu S, Xiao Y and Chen J: MicroRNA-29s could target AKT2 to inhibit gastric cancer cells invasion ability. Med Oncol 32: 342, 2015.

11. Zhang R, Li F, Wang W, Wang X, Li S and Liu J: The effect of antisense inhibitor of miRNA $106 \mathrm{~b} 25$ on the proliferation, invasion, migration, and apoptosis of gastric cancer cell. Tumour Biol 37: 10507-10515, 2016.

12. Xiang XJ, Deng J, Liu YW, Wan LY, Feng M, Chen J and Xiong JP: MiR-1271 Inhibits Cell Proliferation, Invasion and EMT in Gastric Cancer by Targeting FOXQ1. Cell Physiol Biochem 36: 1382-1394, 2015.

13. Zhou X, Xia Y, Li L and Zhang G: MiR-101 inhibits cell growth and tumorigenesis of Helicobacter pylori related gastric cancer by repression of SOCS2. Cancer Biol Ther 16: 160-169, 2015.

14. Zhou X, Xu G, Yin C, Jin W and Zhang G: Down-regulation of miR-203 induced by Helicobacter pylori infection promotes the proliferation and invasion of gastric cancer by targeting CASK. Oncotarget 5: 11631-11640, 2014.

15. Fassan M, Saraggi D, Balsamo L, Cascione L, Castoro C, Coati I, De Bernard M, Farinati F, Guzzardo V, Valeri N, et al: Let-7c down-regulation in Helicobacter pylori-related gastric carcinogenesis. Oncotarget 7: 4915-4924, 2016.

16. Li Q, Wang N, Wei H, Li C, Wu J and Yang G: miR-24-3p Regulates Progression of Gastric Mucosal Lesions and Suppresses Proliferation and Invasiveness of N87 Via Peroxiredoxin 6. Dig Dis Sci 61: 3486-3497, 2016.

17. Yan X, Zhu Z, Xu S, Yang LN, Liao XH, Zheng M, Yang D, Wang J, Chen D, Wang L, et al: MicroRNA-140-5p inhibits hepatocellular carcinoma by directly targeting the unique isomerase Pin1 to block multiple cancer-driving pathways. Sci Rep 7: 45915, 2017.

18. Yu L, Lu Y, Han X, Zhao W, Li J, Mao J, Wang B, Shen J, Fan S, Wang L, et al: microRNA -140-5p inhibits colorectal cancer invasion and metastasis by targeting ADAMTS5 and IGFBP5. Stem Cell Res Ther 7: 180, 2016.

19. Su Y,Xiong J,Hu J, Wei X,Zhang X and Rao L: MicroRNA-140-5p targets insulin like growth factor 2 mRNA binding protein 1 (IGF2BP1) to suppress cervical cancer growth and metastasis. Oncotarget 7: 68397-68411, 2016.

20. Fang Z, Yin S, Sun R, Zhang S, Fu M, Wu Y, Zhang T, Khaliq J and Li Y: miR-140-5p suppresses the proliferation, migration and invasion of gastric cancer by regulating YES1. Mol Cancer 16: 139, 2017.

21. Zou J and Xu Y: MicroRNA-140 Inhibits Cell Proliferation in Gastric Cancer Cell Line HGC-27 by Suppressing SOX4. Med Sci Monit 22: 2243-2252, 2016.

22. Song $\mathrm{Z}, \mathrm{Wu} \mathrm{Y}$, Yang J, Yang D and Fang X: Progress in the treatment of advanced gastric cancer. Tumour Biol 39: 1010428317714626,2017

23. Zhang K, Peng Z, Huang X, Qiao Z, Wang X, Wang N, Xi H, Cui J, Gao Y, Huang X, et al: Phase II Trial of Adjuvant Immunotherapy with Autologous Tumor-derived Gp96 Vaccination in Patients with Gastric Cancer. J Cancer 8: 1826-1832, 2017.

24. Mimura K, Teh JL, Okayama H, Shiraishi K, Kua LF, Koh V, Smoot DT, Ashktorab H, Oike T, Suzuki Y, et al: PD-L1 expression is mainly regulated by interferon gamma associated with JAK-STAT pathway in gastric cancer. Cancer Sci 109: 43-53, 2018.

25. Dos Santos Fernandes G, da Motta Girardi D, Dib Batista Bugiato Faria L, Giacomini Bernardes JP and de Almeida Coudry R: Impressive response to immunotherapy in a metastatic gastric cancer patient: Could somatic copy number alterations help patient selection? J Immunother Cancer 5: 84, 2017.

26. Goodman A, Patel SP and Kurzrock R: PD-1-PD-L1 immune-checkpoint blockade in B-cell lymphomas. Nat Rev Clin Oncol 14: 203-220, 2017. 
27. Salmaninejad A, Valilou SF, Shabgah AG, Aslani S, Alimardani M, Pasdar A and Sahebkar A: PD-1/PD-L1 pathway: Basic biology and role in cancer immunotherapy. J Cell Physiol: Feb 19, 2019 (Epub ahead of print).

28. Liu Y, Wu L, Tong R, Yang F, Yin L, Li M, You L, Xue J and Lu Y: PD-1/PD-L1 Inhibitors in Cervical Cancer. Front Pharmacol 10 65, 2019.

29. Pawłowska A, Suszczyk D, Okła K, Barczyński B, Kotarski J and Wertel I: Immunotherapies based on PD-1/PD-L1 pathway inhibitors in ovarian cancer treatment. Clin Exp Immunol 195: 334-344, 2019

30. Ji X, Wang E and Tian F: MicroRNA-140 suppresses osteosarcoma tumor growth by enhancing anti-tumor immune response and blocking mTOR signaling. Biochem Biophys Res Commun 495: 1342-1348, 2018.

31. McNicholl AG, Ducons J, Barrio J, Bujanda L, Forné-Bardera M, Aparcero R, Ponce J, Rivera R, Dedeu-Cuso JM, Garcia-Iglesias P, et al; Helicobacter pylori Study Group of the Asociación Española de Gastroenterología (AEG): Accuracy of the Ultra-Rapid Urease Test for diagnosis of Helicobacter pylori infection. Gastroenterol Hepatol 40: 651-657, 2017.

32. Livak KJ and Schmittgen TD: Analysis of relative gene expression data using real-time quantitative PCR and the 2(-Delta Delta C(T)) Method. Methods 25: 402-408, 2001.

33. Bayne K; Revised Guide for the Care and Use of Laboratory Animals available. American Physiological Society. Physiologist 39: 199, 208-211, 1996.

34. Kleffel S, Posch C, Barthel SR, Mueller H, Schlapbach C, Guenova E, Elco CP, Lee N, Juneja VR, Zhan Q, et al: Melanoma cell-intrinsic PD-1 receptor functions promote tumor growth. Cell 162: 1242-1256, 2015.
35. Cortez MA, Anfossi S, Ramapriyan R, Menon H, Atalar SC, Aliru M, Welsh J and Calin GA: Role of miRNAs in immune responses and immunotherapy in cancer. Genes Chromosomes Cancer 58: 244-253, 2019

36. Wang Q, Lin W, Tang X, Li S, Guo L, Lin Y and Kwok HF The Roles of microRNAs in Regulating the Expression of PD-1/PD-L1 Immune Checkpoint. Int J Mol Sci 18: 18, 2017.

37. Kapodistrias N, Bobori C and Theocharopoulou G: MiR-140-3p Downregulation in Association with PDL-1 Overexpression in Many Cancers: A Review from the Literature Using Predictive Bioinformatics Tools. Adv Exp Med Biol 988: 225-233, 2017 (In German).

38. Kao SC, Cheng YY, Williams M, Kirschner MB, Madore J, Lum T, Sarun KH, Linton A, McCaughan B, Klebe S, et al: Tumor Suppressor microRNAs Contribute to the Regulation of PD-L1 Expression in Malignant Pleural Mesothelioma. J Thorac Oncol 12: 1421-1433, 2017.

39. Jia L, Xi Q, Wang H, Zhang Z, Liu H, Cheng Y, Guo X, Zhang J, Zhang Q, Zhang L, et al: miR-142-5p regulates tumor cell PD-L1 expression and enhances anti-tumor immunity. Biochem Biophys Res Commun 488: 425-431, 2017.

40. Cioffi M, Trabulo SM, Vallespinos M, Raj D, Kheir TB, Lin ML, Begum J, Baker AM, Amgheib A, Saif J, et al: The miR-25-93-106b cluster regulates tumor metastasis and immune evasion via modulation of CXCL12 and PD-L1. Oncotarget 8: 21609-21625, 2017.

41. Xu S, Tao Z, Hai B, Liang H, Shi Y, Wang T, Song W, Chen Y, OuYang J, Chen J, et al: miR-424(322) reverses chemoresistance via T-cell immune response activation by blocking the PD-L1 immune checkpoint. Nat Commun 7: 11406, 2016. 